\title{
Observation of the Energy Dependence of Primary and Sec- ondary Cosmic Rays with the AMS Detector on the International Space Station
}

\author{
V. Formato ${ }^{1, a}$ \\ on behalf of the AMS-02 collaboration \\ ${ }^{1}$ INFN - Sezione di Perugia, Via A. Pascoli s.n.c., 06123 Perugia (PG)
}

\begin{abstract}
Precision study of cosmic nuclei provides detailed knowledge on the origin and propagation of cosmic rays. AMS is a multi-purpose high energy particle detector designed to measure and identify cosmic ray nuclei with unprecedented precision. It is able to provide precision studies of nuclei simultaneously to multi-TeV energies. In 7 years on the Space Station, AMS has collected more than 120 billion both primary and secondary cosmic rays. Primary cosmic rays, such as $\mathrm{p}, \mathrm{He}, \mathrm{C}$ and $\mathrm{O}$, are believed to be mainly produced and accelerated in supernova remnants, while secondary cosmic rays, such as $\mathrm{Li}, \mathrm{Be}$ and $\mathrm{B}$ are thought to be produced by collisions of heavier nuclei with interstellar matter. Primary cosmic rays such as $\mathrm{He}, \mathrm{C}$, and $\mathrm{O}$ are found to have identical rigidity dependence, similarly to secondary cosmic rays (such as $\mathrm{Li}, \mathrm{Be}$ and $\mathrm{B}$ ) which share the same the same spectral shape. The peculiar case of Nitrogen being a mixture of a primary and secondary component will also be shown.
\end{abstract}

\section{Introduction}

Cosmic rays (CRs) are charged particles coming isotropically from outer space to the Earth atmosphere with an energy range spanning over several decades. It is still unclear where and how CRs are accelerated in the Galaxy, as well as how they propagate through the interstellar medium (ISM), and both these aspects are subjects of current research. Another fundamental aspect in CR physics is the search for any signal of new physics in the antimatter component of CRs that could be linked to the decay of dark matter (DM) particles or to the existence of primordial antimatter. The bulk of cosmic rays is made of protons $(\sim 88 \%)$ and helium nuclei $(\sim 9 \%)$, while the remaining fraction is composed mostly of electrons $(\sim 2 \%)$ and heavier nuclei $(\sim 1 \%)$ with small traces of antimatter (positrons and antiprotons). Depending on wheter a cosmic ray reaches the solar system after being accelerated at its source or after being created in a anelastic interaction of other CRs with the ISM, it is labelled a primary or a secondary cosmic ray. Examples of primary species are protons, ${ }^{4} \mathrm{He}$ nuclei, carbon and oxygen nuclei, and electrons, while typical examples of secondary species are ${ }^{2} \mathrm{H},{ }^{3} \mathrm{He}$, lithium, beryllium and boron nuclei.

Being created directly during the propagation of primary CRs in the Galaxy, secondary CRs carry important information on how the transport process modifies the original CR energy spectrum after the

ae-mail: valerio.formato@pg.infn.it 
acceleration at the source. This is important for the theoretical prediction of the amount of secondary antimatter produced by CR propagation in the Galaxy, since this represents the natural background in the search for new physics in the antimatter channel. Thus the hadronic component of CRs holds valuable information on how CRs are accelerated and on how their energy spectrum is affected by the propagation in the galactic magnetic field. By measuring also secondary species, important informations can be obtained about CR propagation, especially when the flux of a secondary species is divided by the flux of a primary species, preferably one of its progenitors.

Not counting protons, the most abundant species in the hadronic component of CRs is helium, followed by carbon and oxygen wich make up the bulk of the primary component. The secondary component is made up by boron, lithium and beryllium nuclei. Nitrogen defies a clear classification as primary or secondary, as it is supposed to be an admixture of both a primary component and a secondary component.

Light nuclei in CRs with charge $Z \leq 8$ have been recently measured by the AMS-02 collaboration in Refs. [1-3] and the results will be summarized in section 3 .

\section{The AMS-02 experiment}

AMS-02 is a general-purpose high-energy physics particle detector operating onboard the International Space Station (ISS) since May 2011. The instrument will be active for the entire ISS lifetime which is currently forseen to last until 2024. With such a long exposure time and its large geometrical acceptance $\left(\sim 0.5 \mathrm{~m}^{2} \mathrm{sr}\right)$, AMS-02 is capable to provide high-quality data on CR fluxes at the TeV energy scale with unprecedent precision and sensitivity.

The AMS-02 detector is described in details in ref. [4]. It is composed by several sub-detector systems that allow precise particle identification as well as redundant measurements of the main characteristics of CR particles, such as arrival direction, charge and magnetic rigidity $(R=p c / Z e)$. The particle trajectory in the AMS-02 magnetic field is reconstructed from the position measurements along the 9 silicon layers of the tracking system, which provides a spatial resolution of $10 \mu \mathrm{m}$ in the $y$ (bending) view and $30 \mu \mathrm{m}$ in the $x$ (non-bending) view. From the reconstructed trajectory the particle direction and rigidity can be measured. The velocity $\beta=v / c$ can be determined from the transit time between the upper and lower TOF scintillator planes (with a resolution, for $Z=1, \Delta \beta / \beta^{2} \sim 2 \%$ ), or more precisely using the RICH sub-detector (with a resolution, for $Z=1, \Delta \beta / \beta \sim 10^{-3}$ ). The central part of AMS-02 is surrounded by an anti-coincidence system (ACC). The detector is completed with a Transition Radiation Detector (TRD), which is located between the first layer of the silicon tracker and the upper TOF, and a 18-layer electromagnetic calorimeter (ECAL), which is placed at the bottom.

\section{Data analysis}

The key elements used in this measurement are the permanent magnet, the silicon tracker, and the four planes of time of flight (TOF) scintillation counters. The dataset used corresponds to the first 60 months of AMS data taking, from May $19^{\text {th }} 2011$ to May $26^{\text {th }} 2016$, during which AMS recorded $8.5 \times 10^{10}$ cosmic ray triggers. Events of a given species were required to be downward going and to have a reconstructed track in the inner tracker which passes through the first tracker layer (L1). In the highest rigidity region, $R \leq 1.13 \mathrm{TV}$, the track is also required to pass through the last tracker layer (L9). Track fitting quality criteria such as a $\chi^{2} /$ d.o.f. $<10$ in the bending coordinate are applied, similar to [5-7].

Charge measurements on L1, the inner tracker, the upper TOF, the lower TOF, and, for $R>$ $1.13 \mathrm{TV}, \mathrm{L} 9$ are required to be compatible with charge $Z=2$ to 8 for helium to oxygen events 


\begin{tabular}{lcc} 
& N. of events & $\begin{array}{c}\text { Residual background } \\
\text { at } 2 \mathrm{GV} / 3.3 \mathrm{TV}\end{array}$ \\
\hline Helium & $90 \times 10^{6}$ & $<0.1 \%$ \\
\hline Lithium & $1.9 \times 10^{6}$ & $5 \% / 2 \%$ \\
\hline Beryllium & $0.9 \times 10^{6}$ & $8 \% / 13 \%$ \\
\hline Boron & $2.6 \times 10^{6}$ & $5 \% / 8 \%$ \\
\hline Carbon & $8.6 \times 10^{6}$ & $<0.5 \%$ \\
\hline Nitrogen & $2.2 \times 10^{6}$ & $<3 \% / 6 \%$ \\
\hline Oxygen & $7.0 \times 10^{6}$ & $<0.5 \%$ \\
\hline
\end{tabular}

Table 1. Number of events for each species after background subtraction, the amount of residual background subtracted.

respectively. The number of events obtained after background subtraction [1-3] is reported in table 1. With this selection, the charge confusion from non-interacting nuclei is negligible over the whole rigidity range. The residual background comes from heavier nuclei which interact above the tracker L2. The background resulting from interactions in the material between L1 and L2 (TRD and upper TOF) is evaluated by fitting the charge distribution of the tracker L1 charge with charge distribution templates of $\mathrm{Li}, \mathrm{Be}, \mathrm{B}, \mathrm{C}, \mathrm{N}$ and $\mathrm{O}$. Furthermore an additional cut on the $\mathrm{L} 1$ charge is applied as explained in [1-3].

The background from interactions on materials above the tracker L1 (a thin support structures made by carbon fiber and aluminum honeycomb) has been estimated from simulation using MC samples generated according to the AMS flux measurements as detailed in [7]. The estimated residual background levels at $2 \mathrm{GV}$ and $3.3 \mathrm{TV}$ are shown in table 1 . The bin-to-bin migration of events was corrected using the unfolding procedure described in Ref. [6]. A detailed description of the systematic uncertainties can be found in Refs. [1-3].

\section{Conclusions}

The fluxes obtained are shown in Fig. 1 as a function of rigidity with the total errors, the sum in quadrature of statistical and systematic errors. As seen, cosmic ray species can be divided into two distinct families, primary CRs exhibiting a $R^{-2.7}$ power-law behaviour below $300 \mathrm{GV}$, and secondary CRs with a $R^{-3.1}$ power-law behaviour below $300 \mathrm{GV}$. Besides a different normalisation, all primaries fluxes seem to have the same rigidity shape in common, and all the secondaries flux seem to share the same rigidity dependence although different from the primary shape. Remarkably all the species show a deviation from the single power-law shape at $\sim 200 \mathrm{GV}$. This can be also seen examining the evolution of the spectral index as a function of rigidity, shown in Fig. 2. The nitrogen flux is the only one not following either of the two behaviors but, as shown in Ref. [3], it can be well described by a mixture of a primary component and a secondary component both following the spectral shape of the primary and secondary elements respectively.

\section{References}

[1] Aguilar M. et al., Phys. Rev. Lett. 119251101 (2017)

[2] Aguilar M. et al., Phys. Rev. Lett. 120021101 (2018)

[3] Aguilar M. et al., Phys. Rev. Lett. 121051103 (2018) 


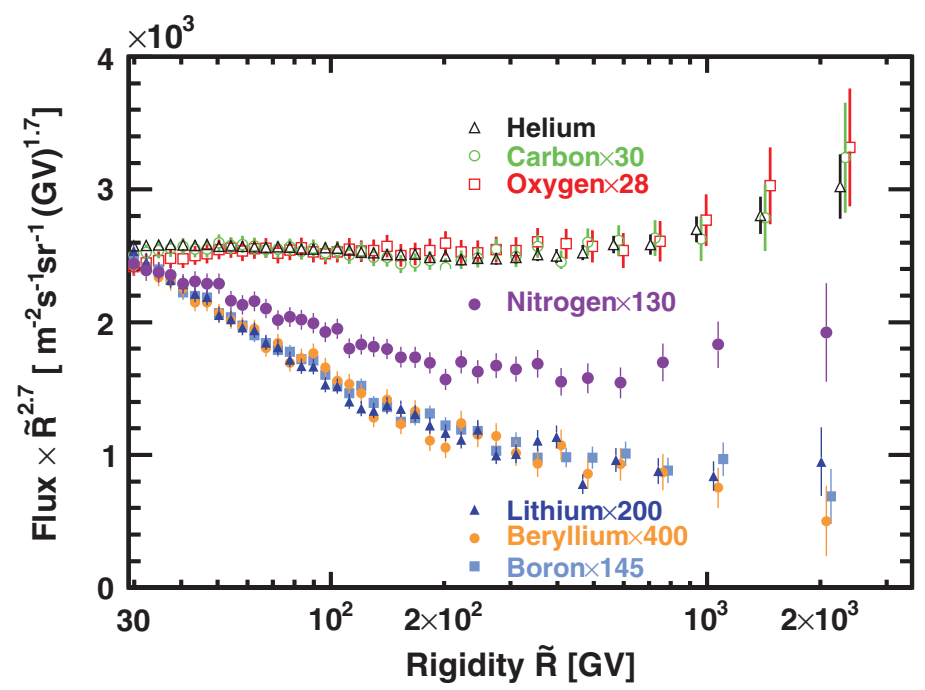

Figure 1. Fluxes of elements from helium to oxygen as measured by the AMS-02 experiment, multiplied by $R^{2.7}$. Each flux is scaled to better show the similar shape of primary and secondary species.

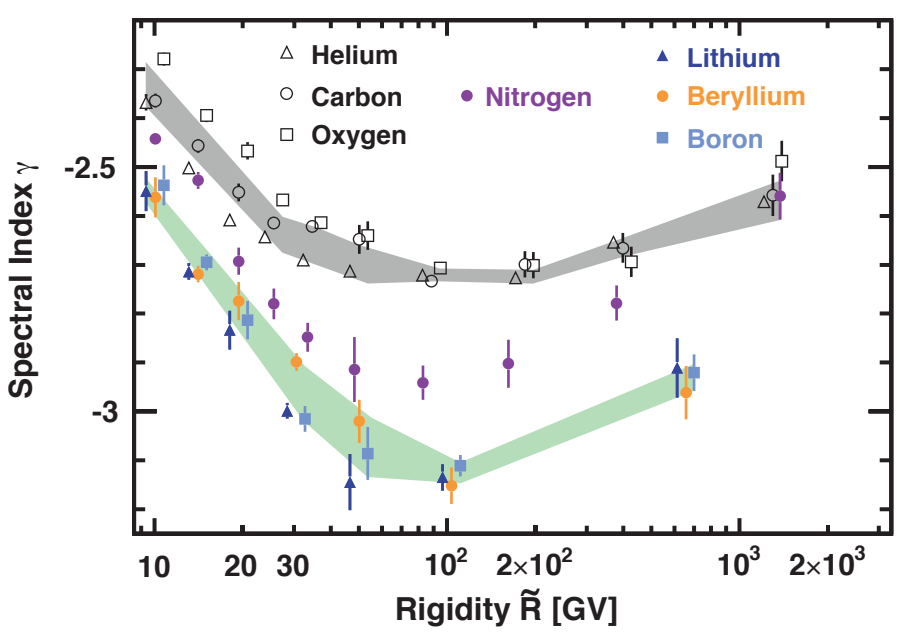

Figure 2. Spectral indices of elements from helium to oxygen as measured by the AMS-02 experiment.

[4] S. Ting, Nucl. Phys. B, Proc. Suppl. 243-244, 12 (2013)

[5] Aguilar M. et al., Phys. Rev. Lett. 115211101 (2015)

[6] Aguilar M. et al., Phys. Rev. Lett. 114171103 (2015)

[7] Aguilar M. et al., Phys. Rev. Lett. 117231102 (2016) 